\title{
Gastroenterologists should wear greens
}

\author{
NOEl B HershField, MD, FRCPC, FACP
}

$\mathrm{O}^{\prime}$ NE OF THE ADVANTAGES OF ADVANCING AGE IS THE ability to look back with, one hopes, a sense of perspective. The advances in our subspecialty of gastroenterology have been so astounding that we have taken them for granted, and so do, it seems, our colleagues and the paying agencies.

My reason for saying this stems from the fact that in the province of Alberta the fee for endoscopy has recently been slashed by $30 \%$, with nary a whisper from our associates in internal medicine or surgery. They, of course, will also have to face such cuts in the future if $\mathrm{I}$ correctly read the tea leaves of medical economics.

Endoscopy is such an easy skill, say our detractors, that it simply is not worth the money. Just like skiing or playing hockey - it is easy if you know how. There has been no comment by them about its accuracy and the ease in which previously suspected diagnoses are ruled in or out. No comment about the fact that when some of us began to realize its potential in the management of upper gastrointestinal bleeding, the blind gastrectomy died a quick death. No comment concerning the fact that without it, new ulcer therapy could not have been evaluated so quickly - and leading to the demise of ulcer surgery, to the situation now that few surgical residents have ever seen or done one!

This fee thing is based on something the economists cooked up called the 'relative value guide'. What is the value of an accurate endoscopy versus suggestive but not diagnostic $\mathrm{x}$-ray? or an inaccurate history, for that matter? How does one compare the management of a sick diabetic against a tough papillotomy?

The answer is that it cannot be done, but our fees commit-

Dr Hershfield is Clinical Professor at the Department of Medicine, University of Calgary, 711 South Tower, 3031 Hospital Drive NW. Calgary, Alberta T2N 2T9 tee tries, as they fight like cats and dogs over the global pot, and end up denigrating each other's skills, whether they be technical or cognitive. Those economists are smart cookies divide and conquer.

That is why we should wear greens and, for added impact, shoes stained with blood, and maybe some feces as well. We should do one of our procedures at night, and then walk around the hospital in sweaty languor, thus enriching our present mundane image. Then talk about relative values. Talk about the fact that because of our efforts the portal-caval shunt has been virtually relegated to the ash heap of history (along with the long complicated postoperative period, and the horrendous complications). Point out to the health care economists that due to our efforts bile duct exploration is becoming a rare bird as well - with uncounted savings to the system, shorter or no hospitalization, and far less morbidity. While we are at it, do not forget the fact that colon polyp removal is almost totally a gastroenterological procedure, mostly done in the outpatient department, and almost without complications. In fact, the history of a myriad of surgical operations are now obsolete: feeding gastrostomy, hiatal hernia repair, cecostomy - just to name the more obvious ones.

The efforts of gastroenterological physicians and researchers have truly changed the face of the diagnosis and therapy of gut troubles. Why then are we the Rodney Dangerfields of internal medicine? So put on your greens - and maybe the tide will change. Do we have to change our appellation to 'cognitive endoscopic gut surgeons', and attack the surgical fee schedule - why not the same fee for papillotomy as surgical common duct exploration? It's cheaper, as effective, and surely as safe.

Gastroenterologists unite - you have nothing to lose but your shirts and ties! 


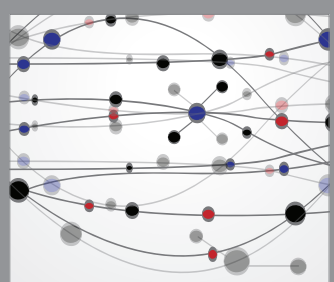

The Scientific World Journal
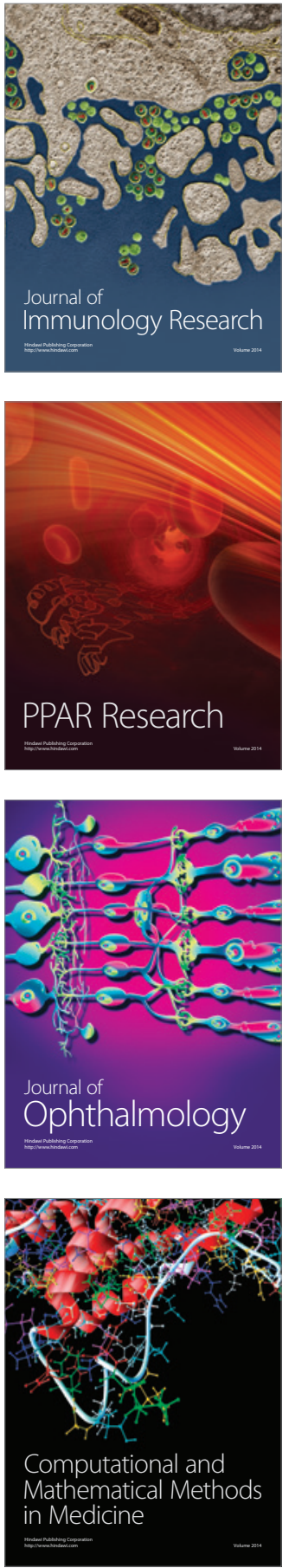

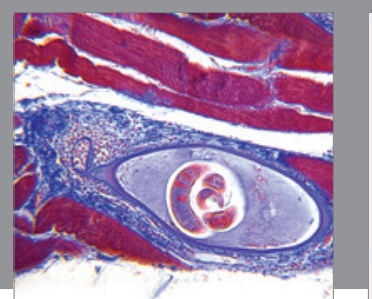

Gastroenterology Research and Practice

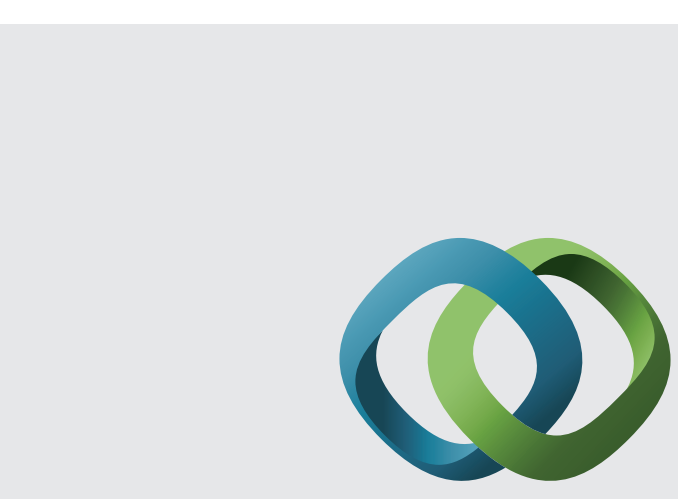

\section{Hindawi}

Submit your manuscripts at

http://www.hindawi.com
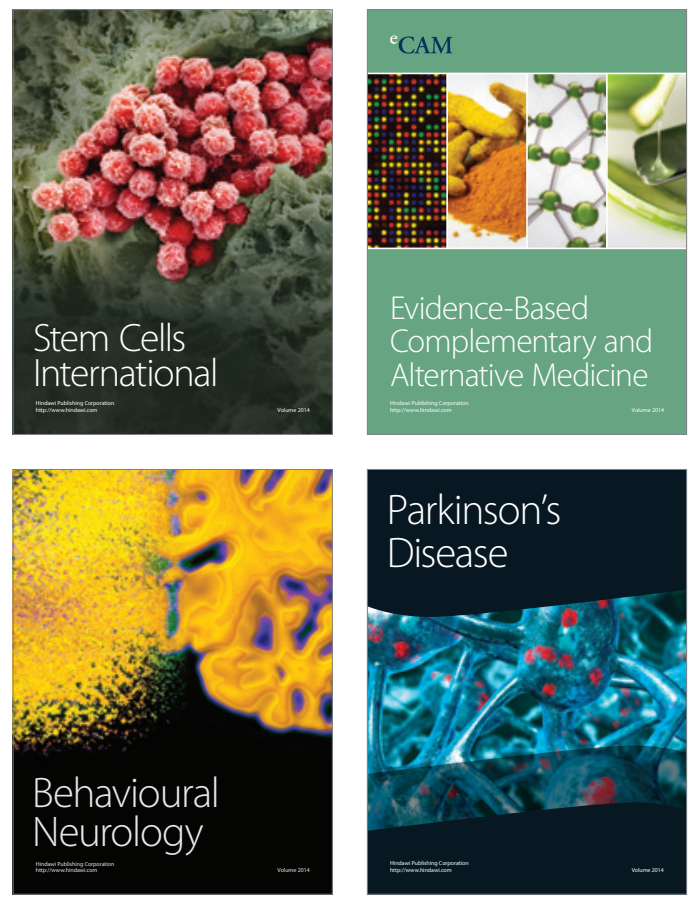
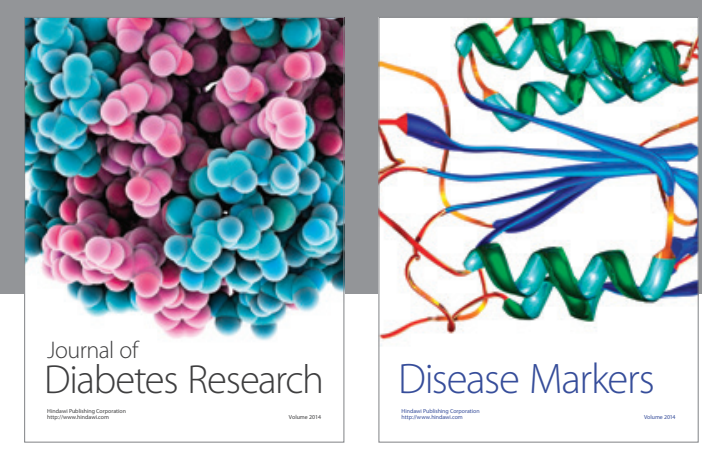

Disease Markers
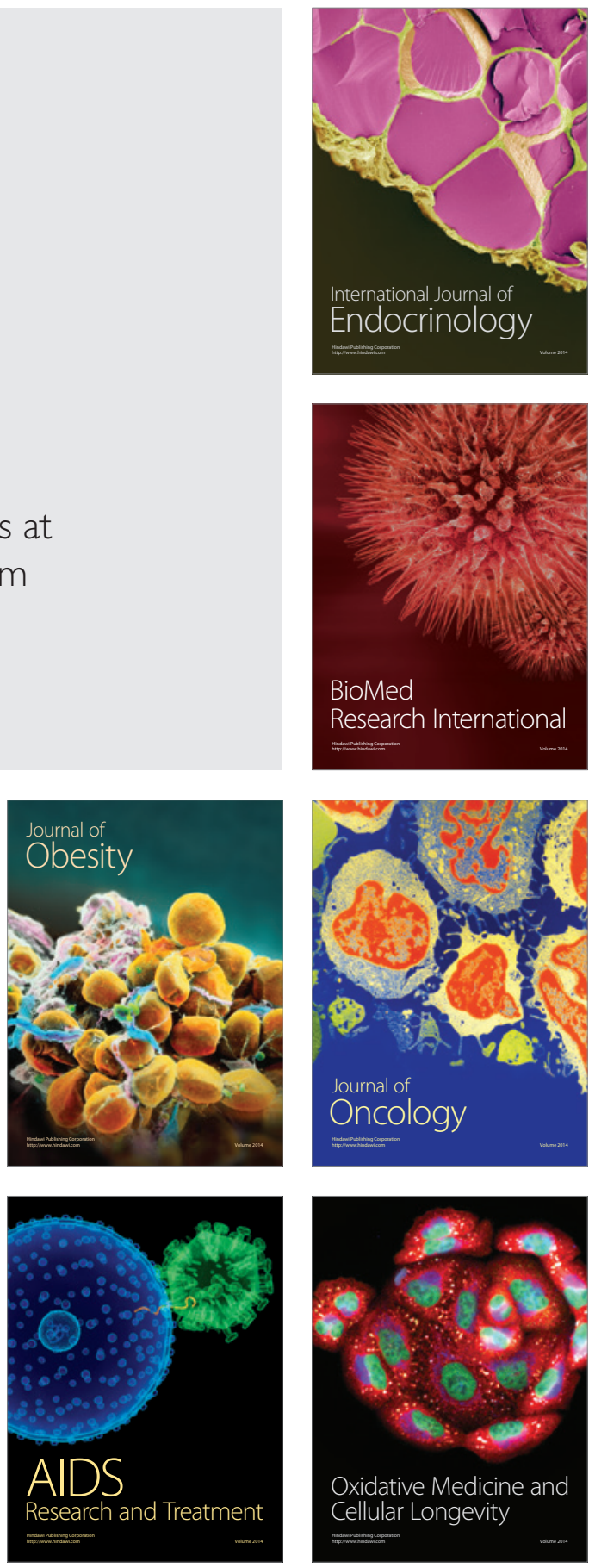